\title{
Europa als „Strahlenbündel nationaler Kräfte“. Zur Konzeption und Legitimation einer europäischen Zusammenarbeit auf der Gründungsfeierlichkeit des „Europäischen Jugendverbandes“ 1942
}

\section{Christoph KÜHBERGER}

Es stellt sich im Zusammenhang mit dem nationalsozialistischen Deutschland die anhaltende Frage nach der politischen Konzeption Europas. Da von offizieller Seite des Regimes nie eine eindeutige Doktrin ausgegeben wurde, werden unterschiedliche politische Aktivitäten meist als anti-europäisch klassifiziert, da bei ihnen die Grundlagen des Rassismus und der Lebensraumideologie nicht überwunden wurden, die letztlich das damalige Europa mitsamt seiner ,geistigen Essenz“" zerstört hätte. ${ }^{1}$ Dabei darf jedoch nicht übersehen werden, dass es durchaus auch andere nationalsozialistische Versuche gab, die nicht nur auf ministerieller Ebene, in den Vorzimmern zur Macht oder in eingeweihten Kreisen durchdacht wurden, sondern vor aller Augen und unter reger Anteilnahme der deutschen Presse. Als ein solches Beispiel gilt die Gründung des „Europäischen Jugendverbandes“ 1942 in Wien. Die bisherige Forschung zu Fragen der europäischen Integration unter dem NS-Regime erwähnt dieses Ereignis zwar, präsentiert jedoch keinen vertieften Blick auf das dort zelebrierte Europaverständnis. Der vorliegende Beitrag versucht daher nach einer knappen Skizze zum Europagedanken im Nationalsozialismus, die Entstehungsgeschichte des Verbandes darzustellen, um schlussendlich der Frage nach der Legitimation eines europäischen Verbandes in einem - in der heutigen Forschung stets betonten - anti-europäischen Klima nachzugehen.

Angesichts des Fehlens eines politischen Grundsatzpapiers in der archivarischen Überlieferung in Wien und Berlin können die politischen Visionen und Grundpfeiler des „Europäischen Jugendverbandes“ nur über Umwege erschlossen werden. Zugänge zur inhaltlichen Konzeption und zur Struktur der Zusammenarbeit auf europäischer Ebene werden daher hier über Presseberichte aus dem In- und Ausland, über den Aktenbestand für die Planung und Umsetzung der Gründungsfeierlichkeiten, die im Archiv der Republik in Wien aufbewahrt werden, sowie über die offiziellen Reden und Publikationen der Veranstalter selbst gelegt.

1. M. Salewski, Ideas of the National Socialist Government and Party, in: W. LiPgens, Documents on the History of European Integration, Walter de Gruyter, Berlin, 1985, S 37-54, S.54. 


\section{Nationalsozialismus und Europagedanke}

Wird der Nationalsozialismus als radikaler und rassistisch übersteigerter Nationalismus verstanden, wie dies in den meisten Interpretationen des NS-Regimes der Fall ist, fällt es schwer danach zu fragen, worin die europäische Dimension der nationalsozialistischen Herrschaftskonzeption bestand. Zudem entwickelte der Nationalsozialismus keine genuine europäische Idee oder verfolgte eine konkrete europäische Politik. Vielmehr blockierten die Blut- und Boden-Mythologie und die Rassenideologie den Transfer des nationalsozialistischen Herrschaftskonzeptes auf andere Kulturen. $^{2}$

Die nationalsozialistische Politikerriege interessierte sich nur vage für Europa. Deutschland gelesen als „Großdeutsches Reich“ stand lange im alleinigen Fokus. Europa als Raum wurde erst viel später aktiv in politische Strategien des Regimes eingebunden. Es verwundert daher auch nicht, dass Alfred Rosenberg als einer der wichtigsten Ideologen des Nationalsozialismus sich dem Thema als einer der wenigen stellte, ohne jedoch in den breiten offiziellen Diskurs Eingang zu finden. Rosenberg identifizierte vor allem ein Europa der Einzelnationen und ignorierte dabei die bereits vorhandene Diskussion aus der Zwischenkriegszeit, in der Europa mit Friedenssicherung, Überwindung der Wirtschaftskrise durch eine Wirtschafts- und Zollunion o.ä. in Verbindung gebracht wurde. ${ }^{3} 1934$ hielt Rosenberg in einem Vortrag fest:

„Der Punkt, die Idee, die Tatsache, von der wir alle ausgehen müssen, ist die Tatsache der Nation. Der Nationalismus ist [...] heute lebendiger als jemals in früheren Jahrhunderten [...], er zeigt ein vielgestaltiges Gesicht. Diese Vielgestaltigkeit hat ihre Wurzeln in ganz bestimmten Volkscharakteren. Ich glaube, daß die Art und Weise, wie ein Volk seine Idee des Nationalismus prägt, die entscheidende kulturgeschichtliche und politische Tatsache europäischer Geschichte darstellt". ${ }^{\text {}}$

Rosenberg positionierte in diesem Vortrag jedoch auch den Antikommunismus, den Rassegedanken, den Antidemokratismus, den Kolonialismus und hebt den Stellenwert der Grenze hervor. ${ }^{5}$ Eine europäische Integration wurde, wie oben skizziert, in den ersten Jahren der NS-Herrschaft seitens der Nationalsozialisten nicht diskutiert. Man versuchte vielmehr all jene Traditionen zu unterbinden, die den doktrinären Selbstverständnis der NSDAP zuwider liefen, weshalb etwa ab der Machtergreifung 1933 alle europäischen Vereinigungen in Deutschland sofort als pazifistisch verboten

2. M. GEHLER, Europa. Ideen, Institutionen, Vereinigung, Olzog, München, 2005, S.111 f.

3. W. SCHMALE, Geschichte Europas, Böhlau, Wien/Köln, 2000, S.116; M. SALEWSKI, op.cit., S. 37.

4. Rosenbergs Ausführungen stammen aus dem Vortrag „Krisis und Neubau Europas“ (1934) - Zitiert nach W. SCHMALE, op.cit., S.115.

5. G. SIMON, Europa-Gedanke und Sprachpolitik 1933-1945, in: http://homepages.uni-tuebingen.de/ gerd.dimon/EuroMarbg.pdf(20.05.2009); Vgl. auch G. SIMON, Europa-Gedanke und Sprachpolitik 1933 bis 1945, in: INSTITUT FÜR AUSLANDSBEZIEHUNGEN (Hrsg.), Sprachenpolitik in Europa-Sprachenpolitik für Europa (= Materialien zum Internationalen Kulturaustausch Bd. 36), Ifa, Stuttgart, o.J. (1997), S.39-45. 
wurden. ${ }^{6}$ Erst der Zweite Weltkrieg führte zu einer verstärkten Auseinandersetzung mit Europa als Raum. Der Krieg und die anfänglichen deutschen Siege setzen angesichts der gewaltsamen Vereinheitlichung Europas unter nationalsozialistischer Herrschaft ein Denken in kontinentalen Zusammenhängen auf die politische Agenda. Am spürbarsten wurde die „europäische Dimension“ zuerst im wirtschaftlichen Bereich, wo Göring im Juni 1940 im Rahmen der Überlegungen zum Vier-Jahres-Plan eine neue europäische Wirtschaftsordnung auf den Weg brachte. ${ }^{7}$ Gleichzeitig verkörperte der Nationalsozialismus

„für eine nicht unerhebliche Zahl von Intellektuellen [in den besetzen Ländern Westeuropas] den Fortschritt, der mit Waffen einen großen zukunftsfähigen Wirtschaftsraum Europa schaffe, ein europäisches Europa in Bewegung; sie träumten von einer europäischen Wiedergeburt, einer europäischen Revolution, einer europäischen faschistischen Zivilisation, von einem schöpferischen und vitalen kontinentalen Block". 8

Die NS-Propaganda bediente gerne derartige Visionen. Aus machtstrategischen und propagandistischen Erwägungen heraus entwickelten die Nationalsozialisten - so Wolfgang Schmale - Europakonzepte, die gemessen an den Traditionen der Europaidee heute eher als anti-europäisch eingestuft werden sollten. ${ }^{9}$

Der Krieg führte so zur Konjunktur des Europäischen. Während auf der einen Seite sich ein demokratischer und ein kommunistischer Widerstand in Europa formierte, feierten Teile der rechts-konservativen, faschistischen bzw. imitationsfaschistischen Strömungen den nationalsozialistischen Siegeszug durch Europa. Aus taktischen Gründen mussten die deutschen Nationalsozialisten von den ursprünglich propagierten germanozentrischen Konzepten Abschied nehmen. Mit Germanen- und Deutschtum konnte der neu eroberte Herrschaftsraum nicht Aufrecht erhalten werden. Selbst Adolf Hitler versuchte vor dem Hintergrund des Kriegseintrittes der USA in einer Rede von 1941 diese notwendige Neuausrichtung auszubalancieren und verwies auf die „europäische Front" bestehend aus Deutschland, Italien, Spanien und Kroatien, die den Kontinent mit Freiwilligen aus Nord- und Westeuropa verteidigen würde. ${ }^{10}$ Hitlers Zugeständnisse müssen jedoch eher als propagandistische Lippenbekenntnisse verstanden werden, denn im engeren Kreis seines Führerkaders machte er auch zu diesem Zeitpunkt unmissverständlich deutlich, dass die bei Rosenberg bereits Anfang der dreißiger Jahre zu findenden Ideologeme nicht überwunden sei-

6. G. BRAUN, Die Europäische Einigung, Reclam, Stuttgart, 2002, S.26.

7. M. SALEWSKI, op.cit., S.42 f.

8. G. BRAUN, Die Europäische Einigung, Reclam, Stuttgart, 2002, S.27.

9. W. SCHMALE, Geschichte und Zukunft der Europäischen Identität, Kohlhammer, Stuttgart, 2008, S.59.

10. Vgl. M. DOMARUS, Hitler. Reden und Proklamationen. 1932-1945, Bd. II/2, Süddeutscher Verlag, München, 1962, S.1796 ff. - Reichstagsrede Hitlers vom 11.12.1941. Michael Salewski sieht im Angriff auf die Sowjetunion (Juni 1941) den Wendepunkt in der Wahrnehmung von Europa als Einheit, wenn auch im nationalsozialistischen Verständnis als Einheit gegen das ,,asiatisch-jüdischbolschewistische“ und anglo-amerikanisch Andere. Vgl. M. SALEWSKI, op.cit., S.48 f. 
en. ${ }^{11}$ Bis zum Untergang des Regimes wurden keine klaren Konzeptionen für die Integration Europas entwickelt. ${ }^{12}$

\section{Auf dem Weg zur Gründung des, „Europäischen Jugendverbandes“}

Baldur von Schirach, der 1942 das Amt des Reichsstatthalters in Wien bekleidete und Reichsleiter der HJ war, gehörte seit Anfang der vierziger Jahre nicht mehr zum engeren Kreis um Hitler. Die Minister Josef Goebbels und Joachim von Ribbentrop arbeiteten gegen ihn und auch Martin Bormann, der in der Wolfsschanze Informationen an Hitler weiterleitete, half mit, Schirachs Einfluss einzudämmen, wodurch der direkte Zugang zu Hitler und andere Kommunikationsmöglichkeiten mit dem Zentrum der nationalsozialistischen Macht erheblich eingeschränkt wurden. ${ }^{13}$ Schirach wurde daher wohl selbst von der propagandistischen Taktik getäuscht, als er dazu ansetzte, den „Europäischen Jugendverband“ zu gründen, der ein europäisches Kollektiv im Sinn der faschistisch-nationalsozialistischen Ideologie stiften sollte. Dies wurde jedoch schlussendlich nicht zuletzt von Hitler selbst unterbunden, „da jede Form von Vernetzung auch die Teilung von Herrschaft erfordert und keine uneingeschränkte Herrschaft, wie sie Hitler wollte, zugelassen hätte“. ${ }^{14}$ Gleichzeitig sollt man jedoch auch Schirachs persönliche Begehrlichkeiten im neuen europäischen Mächtespiel beachten.

„Mit dem deutschen Vormarsch an allen Fronten und der gewaltigen Ausdehnung des nationalsozialistischen Machtbereichs im Sommer 1942 begann auch Schirach in kontinentalen Dimensionen Politik zu betreiben. Auch er wollte seinen Anteil an der europäischen Führungsrolle des Reiches und seinen Einfluss auch auf die Jugendorganisationen der deutschen Satelliten ausdehnen“. ${ }^{15}$

Interpretiert man Schirachs Gründung des „Europäischen Jugendverbandes“ als das Resultat einer fehlgeleiteten parteiinternen Kommunikation, indem er selbst der aufkeimenden Europa-Propaganda, die ursprünglich zur Beschwichtigung der unterworfenen Länder und zur Motivation der Kollaborateure inszeniert wurde, Glauben schenkte, sowie als Profilierungsversuch von Schirach selbst, der sich mit dieser Aktion im Bereich der kontinentalen Politik in den Vordergrund und damit in den Wahr-

11. Vgl. H. PICKER, Hitlers Tischgespräche im Führerhauptquartier, Propyläen, Berlin, 1999³, S.94. - Aufzeichnung vom 08./09.09.1941.

12. W. BURGDORF, ,Chimäre Europa“. Antieuropäische Diskurse in Deutschland (1648-1999), Winkler, Bochum, 1999, S.191. Zur Geschichte der nationalsozialistischen Raumkonzepte, vgl. B. KLETZIN, Europa aus Rasse und Raum. Die nationalsozialistische Idee der Neuen Ordnung, Lit, Münster, 20022; U. MAI, „,Rasse und Raum“. Agrarpolitik, Sozial- und Raumplanung im NSStaat, Schöningh, Paderborn, 2002.

13. Vgl. M. WORTMANN, Baldur von Schirach. Hitlers Jugendführer, Böhlau, Köln, 1982, S.197 ff.; J. von LANG, Der Hitler-Junge. Baldur von Schirach. Der Mann, der Deutschlands Jugend erzog, Rasch u. Röhring, Hamburg, 1988, S.324.

14. W. SCHMALE, Geschichte und Zukunft ..., op.cit., S.60.

15. M. WORTMANN, op.cit., S.209 ff. 
nehmungshorizont Hitlers schieben wollte, ${ }^{16}$ dann kann man verstehen, warum die Bemühungen letztlich erfolglos blieben. Das Projekt konnte nämlich nicht jene vermutlich angestrebte - Durchschlagkraft entwickeln, wie gewollte und konzertierte Aktionen von NSDAP und NS-Staat. Nicht nur der fortgeschrittene Weltkrieg, sondern auch die Gegner Schirachs in der NSDAP verhinderten einen parteiinternen und damit wirksamen politischen Erfolg. Aus Schirachs Perspektive erschien die Gründung eines solchen Verbandes bestehend aus den unterschiedlichen Staatsjugendorganisationen wohl als eine logische Genese des bisherigen Weges der HJ. ${ }^{17}$ Die bilateralen Beziehungen zum faschistischen Italien und seiner Jugendorganisation (G.I.L.) sollten dafür eine bereits bewährte Grundlage bieten. Die beiden Regimes bewunderten den Organisationsgrad der jeweils anderen Organisation. ${ }^{18}$

Dem Gründungstreffen in Wien 1942 waren zahlreiche gemeinsame Aktivitäten vorausgegangen. In der Selbstwahrnehmung der deutschen Organisatoren waren dabei vor allem die Sommerkampfspiele der HJ in Breslau im August 1941 von Bedeutung. Bereits dort beschwor Schirach das europäisch Gemeinsame:

„Gemeinsame Ideale verbinden uns: das Ideal der Selbstführung der Jugend, das Ideal der sozialen Gerechtigkeit und der Gedanke der Schicksalsgemeinschaft der jungen Generation Europas. Wir sind Brüder und Schwestern im Kampf um die Freiheit des europäischen Geistes gegen den Terror des Bolschewismus und gegen die Macht des Goldes. Für diese Ideale marschieren wir und kämpfen wir. [...] Hier in Breslau verstehen wir auch, daß der Streit der eigenen Völker Europas untereinander einmal wesenslos sein wird vor der Gemeinschaft, Einigkeit und Verbundenheit jenes kommenden Europas, das wir in der Jugend heute schon bekennen". 19

Diese Wettkämpfe, an denen bereits 14 Nationen teilnahmen, bewegten Schirach auch dazu, Benito Mussolini telefonisch Grüße auszurichten. ${ }^{20}$ Schirach sah die Achse Berlin-Rom als zentrale Drehscheibe eines neuen Europas an. In seiner Rede „an die Jugend Europas" führte er dazu in Breslau aus:

„Uns allen, die wir in solchem Geist versammelt sind, geht in tiefer Dankbarkeit das Wunder der Wiedergeburt des europäischen Geistes auf, wenn wir dessen gedenken, der heute, gemeinsam mit Benito Mussolini, Schwert und Fahne Europas in den Händen hält"“.21

Bereits bei dieser Gelegenheit sprach Schirach seine Vorstellungen über eine „Arbeitsgemeinschaft der Jugend Europas“ an und dachte dabei an einen „Jugendverband“", in dem ,,alle Jugendorganisationen Europas nach freiem Willen und Ermessen

16. Vgl. J. von LANG, op.cit., S.324. - Hitlers Antwort auf die telegraphischen Grüße von der Gründungsveranstaltung fiel sehr knapp aus und ist wohl als Standardtext zu verstehen, der dem Ereignis keinerlei Bedeutung beimisst. Völkischer Beobachter (VB), Wiener Ausgabe, 17.09.1942, S.1.

17. Archiv der Republik (AdR), Reichsstatthalter (RSTTH) Wien, Kt. 54, 281; Günter Kaufmann: Von Potsdam bis Wien. Die Einigung der deutschen und der europäischen Jugend. - Kt. 53, 278; Presseberichte - vgl. Il Corriere della Sera, 15.09.1942.

18. B. von SCHIRACH, Ich glaubte an Hitler, Mosaik-Verlag, Hamburg, 1967, S.223 f.

19. AdR, RSTTH Wien, Kt. 53, 278. - Schirach an die europäische Jugend, in: Neues Wiener Tagblatt, 29.08.1941, o.S.

20. Vgl. Archivio Centrale di Stato (ACS), SPD, co 525.199.

21. AdR, RSTTH Wien, Kt. 53, 278. - Schirach an die europäische Jugend, op.cit. 
mitarbeiten"sollten. Ein Gedanken, den der italienische Vertreter Orfeo Sellani dankend als zukunftsweisend aufnahm..$^{22}$ Im Mai 1942 wurde dieser Weg einer Internationalisierung auf europäischer Ebene durch eine deutsch-italienische Jugendkulturkundgebung in Weimar unterstrichen. ${ }^{23}$ Diese Überbetonung der Achsenmächte, um die sich die restlichen europäischen Staaten formieren sollten, war zu diesem Zeitpunkt jedoch nicht mehr unhinterfragt.

„The idea of a united Europe under German and Italian leadership was more and more overlaid by the vision of a 'Germanic' or 'Greater Germanic' Reich. This objective, which was that of Himmler and the SS, was often by no means welcome to Goebbels and Rippentrop, who inclined more towards traditional ideas of a continental bloc". ${ }^{24}$

Dies sollte Schirach auch zu spüren bekommen.

Während der Reichssatthalter sich bemühte, für den 14. bis 18. September 1942 die Gründungsfeierlichkeiten und eine daran gekoppelte Tagung in Wien aufgrund der kriegsbedingten Einschränkungen im würdigen Rahmen zu veranstalten, arbeiteten andere dagegen. Außenminister Ribbentrop ließ „das Vorhaben durch die diplomatischen Vertreter des Reiches im Ausland abwerten, und Goebbels wies Presse und Rundfunk an, sich damit nur am Rande zu beschäftigen" ${ }^{25}$ Der italienische Außenminister und Schwiegersohn des „Duce“ Graf Galeazzo Ciano, der sein Kommen bereits zugesichert hatte, zog seine Zusage zurück. ${ }^{26}$ Letztlich konnten auch der ehrwürdige Rahmen im „Gauhaus“, dem früheren österreichischen Parlament, die penibel angelegten Minutenprogramme, die Defilees der HJ und das Kulturprogramm nicht darüber hinwegtäuschen, dass es sich bei der Veranstaltung um ein zweitrangiges Anliegen der NSDAP handelte. Nur Reichsorganisationsleiter Robert Ley, ein Freund Schirachs, war bei der Eröffnung der Veranstaltung anwesend. Weitere höhere parteiliche Würdenträger der Reichsebene fehlten. Ansonsten feierten sich die Staatsjugendführer und die Führerabordnungen von 14 europäischen Nationen vor allem selbst. ${ }^{27}$

Es spricht einiges dafür den „Europäischen Jugendverband“ als eine „Chimäre“ zu klassifizieren, da die vernichtenden militärischen Niederlagen des „Deutschen Reiches" wenige Monate später ihn erst gar nicht zur Entfaltung kommen ließen.28 Aber nicht nur die Wende des Krieges ließ den „Europäischen Jugendverband“ in die Bedeutungslosigkeit fallen, sondern auch eine Verfügung der NSDAP, in der Hitler

22. Bundesarchiv (BA) Berlin, Personalakt Baldur von Schirach. - Baldur von Schirach vor der europäischen Jugend, in: $V B, 30.08 .1941$, o.S..

23. M. WORTMANN, op.cit., S.211.

24. M. Salewski, op.cit., S.40.

25. J. von LANG, op.cit., S.324.

26. M. WORTMANN, op.cit., S.211.

27. Vgl. VB, Wiener Ausgabe, 15.09.1942, S.1. Die beteiligten Nationen waren: Spanien, Italien, Rumänien, Dänemark, Ungarn, Norwegen, Belgien, Kroatien, Niederlande, Finnland, Slowakei, Bulgarien, Portugal und Deutschland. Landes- und Stadtarchiv Wien, MD-PB A 15/2 (1942), Europäischer Jugendverband. - Liste der Teilnehmer an der Gründungstagung des Europäischen Jugendverbandes, Stand vom 12.IX.1942, 15 Uhr. Vgl. auch VB, Wiener Ausgabe, 15.09.1942, S.1.

28. M. WORTMANN, op.cit., S.213. 
die außenpolitischen Kompetenzen klar in die Hand des Reichsaußenministers legte, kritisierte die Gründung eindeutig. Hitler stellte klar:

„Bei der Pflege zwischenstaatlicher Beziehungen durch Parteistellen darf niemals vergessen werden, daß die Grundlagen und Erkenntnisse der nationalsozialistischen Weltanschauung dem Wesen des deutschen Blutes entsprechen und daher auf fremdes Volkstum nicht übertragen werden können. Das Zusammenleben der Völker verlangt gegenseitige taktvolle Rücksichtnahme auf ihre naturgegebenen Eigenarten. Die NSDAP. und ihre Organisationen haben daher keine europäische oder weltumfassende Missionsaufgabe zu erfüllen. Das Gebiet der Außenpolitik ist nicht geeignet zu Experimenten und persönlichen Bestrebungen". 29

Hitler verbat in der Verordnung explizit die Planung, Vorbereitung und Durchführung europäischer oder internationaler Kongresse, Tagungen, Gründungen von Verbänden usw. Aufgrund des engen zeitlichen Zusammenhangs ist es schwerlich zu leugnen, dass damit vor allem Schirachs Bestrebungen in die Schranken gewiesen wurden. Gleichzeitig muss man jedoch festhalten, dass die Zusammenarbeit zwischen dem auf der Gründungsfeier eingesetzten Präsidenten des „Europäischen Jugendverbandes“, Reichsjugendführer Arthur Axmann, und dem Generalsekretär der faschistischen Partei Aldo Vidussoni, trotz der auch in Rom spürbaren Unstimmigkeiten zwischen der HJ und dem Reichsaußenministerium fortgesetzt wurde. ${ }^{30}$

Unabhängig von der weiteren Verbandsgeschichte, die bisher selbst für diese kurze Periode nur unzureichend erforscht ist, stellt die Gründungstagung in Wien 1942 ein Reservoir für nationalsozialistische Europakonzepte dar. Es handelt sich dabei um ein seltenes Beispiel, in dem Europa als Gemeinschaft von Völkern bzw. Nationen (auch über die NS-Presse) in den öffentlichen Diskurs gelangte.

\section{Legitimationsversuche: Europa-Konzept(e) der Gründungsfeierlichkeiten}

Eric Hobsbawm verweist darauf, dass historische Kontinuität von einer neuen Institution erst erfunden werden muss, um jene Legitimität zu erreichen, die andere Institutionen aufgrund von eingeschliffenen Gewohnheiten bereits besäßen. ${ }^{31}$ Bei der Gründung des „Europäischen Jugendverbandes“ unter nationalsozialistischer Ägide wurden dazu unterschiedliche Begründungsmuster herangezogen, die dazu im Stande sein mussten, den bisherig favorisierten und durchgesetzten (radikal) nationalistischen Kurs interpretativ zu überwinden, ohne ihn dabei zu beschädigen. Um diesen Voraussetzungen zu genügen, wurden Versatzstücke aus der Vergangenheit, der Ge-

29. AdR, RSTH Wien, Hauptbüro Schirach, Kt. 378/ Reichsverfügungsblätter. - NSDAP: Reichsverfügungsblätter, Ausgabe A, Folge 45/42, 11.11.1942 - Verfügung V19/42.

30. ACS, Archivio Renato Ricci, b. 4/58. - Vgl. dort das Promemoria per il Duce sowie den Schriftverkehr zwischen Ricci und Axmann.

31. Vgl. E. Hobsbawm, Inventing Traditions, in: E. Hobsbawm, T . Ranger (Hrsg.), The Invention of Traditions, Cambridge University Press, Cambridge 1992. S.1-14, S.2. 
genwart und der Zukunft herangezogen und durch transzendentale Momente, hier zu verstehen als Rückgriff auf das Mythisch-Göttliche, ergänzt.

\title{
Legitimation über die Vergangenheit
}

Eine Möglichkeit, die Legitimation des Verbandes herauszustellen, war der Rekurs auf die (kultur)geschichtlichen Leistungen, die Europa einigen würden. Man konnte dabei nicht auf jene Beispiele zurückgreifen, die unter dem NS-Regime für das nationalsozialistische Geschichtsbewusstsein auch im Lichte eines germanischen Rassismus herausgearbeitet wurden (z.B. Widukind), ${ }^{32}$ sondern musste historische Modelle heranziehen, mit denen sich die Mehrzahl der teilnehmenden Jugendorganisationen aus unterschiedlichen Staaten Europas identifizieren konnten. In der offiziellen Veröffentlichung zur Tagung „Europa - Kontinent der Jugend“, die anlässlich der Gründungsfeierlichkeiten abgehalten wurde, ist dieser Zugang am stärksten spürbar. Zunächst ist es das römische Reich, welches als Vorbild für „erfolgreiche Großraumpolitik auf europäischem Boden“ herangezogen wird. Dass dieses Reich auch Gebiete in Afrika und Asien besaß, wird zwar erwähnt, aber marginalisiert. Vielmehr werden staatliche Organisationsstrukturen vorgestellt, die inhaltlich und strukturell als Abbild und Empfehlung für den Nationalsozialismus gelesen werden können. Zum 3. Jahrhundert merkt etwa Rudolf Egger an:

\begin{abstract}
„Not, und noch einmal Not, war die Signatur dieser Zeit, unendlich viel schwerer der Ausweg zu finden als lediglich bei der politischen Krise am Ende der Demokratie. Den heroischen Kampf nahm ein einzelner auf, der Gardeoberst Diocletian. Er hat dem Reich seine letzte Form gegeben. [...] Die Not sollte durch äußere Opfer gebändigt werden, daher Organisation bis zum letzten und Kontrolle bis zum letzten. Der einzelne sollte nur in Gemeinschaft leben. Das waren die Grundgedanken. Die Landleute wurden schollenpflichtig, die erwerbenden Berufe in Zwangsverbänden gegliedert, die Besitzenden haftbar für das Steuersoll gemacht".
\end{abstract}

Unverblümt endet die Ausführung mit einem Gegenwartsbezug, der im Kontext von 1942 wenig Raum für Interpretationen lässt:

„Das Imperium Romanum ist niemals gestorben, sondern lebt durch die nie wieder erlöschende Sehnsucht der Völker Europas noch einer höheren Einheit im Gedächtnis der abendländischen Menschheit fort. Der Gedanke des römischen Reiches strahlt noch in das Mittelalter aus und heute feiert er im Zeichen der neuen Ordnung unseres Kontinents und seiner Einheitsfront zur Bekämpfung der tödlichen Gefahr im Osten jungendstark seine Auferstehung. Vorbildlich wirke das Imperium für alle Völker wegen der Begründung dauerhafter politischer Formen und wegen der menschengewinnenden Kraft, die so viele und so verschiedene in ihren Bannkreis zog". ${ }^{33}$

32. Vgl. W. Burgdorf, op.cit., S.194 ff.

33. R. EGGER, Europa und das Imperium Romanum, in: Europa - Kontinent der Jugend, Verlag „Die Pause“, Leipzig 1942, S.9-12, hier: S.12. 
Die Darstellung des römischen Reiches aus einer europäischen Perspektive im Jahr 1942 steht exemplarisch für auch andere historische Erörterungen im Tagungsband. Immer wieder wird versucht, das europäisch Gemeinsame zu betonen. Dazu wird etwa auch die Zeit der Türkenkriege herangezogen:

„Weltgeschichtliche Bedeutung aber gewann das Reich [...], als es von neuem Schutz und Schirm Europas gegen die damalige Weltgefahr des osmanischen Reiches wurde. [...] Das Österreich der Barockzeit bedeutet die wichtigste Reichsschöpfung seit den großen Planungen der Staufferzeit. In Josef I. und Prinz Eugen scheinen das Ordnungsprinzip der kaiserlichen Gewalt und die territoriale Erbmasse des Hauses Habsburg, beide deutsch und übervölkisch zugleich in ihren Interessen in die europäische Aufgabenfülle hineinreichend, zu einer unzertrennlichen Einheit zusammenzuwachsen, um aus dem österreichischen Raum heraus ein gesamtdeutsches Gefüge zu schaffen, das sich bis an die Grenzen der ostslawischen Welt erstreckte und im überwölbenden Barockdenken [...] seine geistigen Wurzeln hat. Wenn in einer Epoche das deutsche und das abendländisch-europäische Interesse identisch waren, dann in dem Zeitalter des Prinz Eugen [... $]^{\text {“. } 34}$

Das Gemeinsame ist jedoch nicht nur eine politikgeschichtliche Kategorie, sondern auch eine der Kunst. So wurden im Frühmittelalter ,,wesensverwandte Grundhaltungen des Kunstwillens“ ausgemacht, die vor allem in einer ,ureuropäisch-christlichen Stein- und Holzbildnerei“" ihren gesamteuropäischen Ausdruck finden würden. ${ }^{35}$ Identifiziert wurden dabei als "Grundpfeiler der gesamteuropäischen Kultur" das „griechische Altertum“ und das „nordisch geformte“, besonders das „germanischmittelalterliche Christentum". 36

Doch nicht nur das Gemeinsame als sinnstiftende Einheit wird dargelegt, sondern auch das Trennende, das u.a. durch eurozentrische Positionen eingebracht wird. Anhand von historischen Beispielen werden die ,,anderen“ abgewertet, um die europäische Gemeinschaft zu stärken. So wird etwa in der musikgeschichtlichen Abhandlung von Alfred Orel ein „erster Kampf zwischen Europa und Asien“ beschrieben:

„Über den Süden stoßen Norden und Osten hart aufeinander. Denn mit den orientalischen Weisen des christlichen Ritus drang eine dem nordischen Musikempfinden unverständliche, ihm geradezu zuwiderlaufende Musik in den bisherigen Geltungsbereich einer im technischen wohl weit einfacheren, unmittelbar aus der nordischen Volksseele erwachsenden, durchaus ursprünglichen Kunst".

Nach Orel entstand daraus das Europäische, da das „Klangbewusstsein des Nordens $[\ldots]$ im umfassenden Vordringen geradezu das künstlerische Wesen des aufgezwungenen Kunstgutes" vernichtete und den Einzelklang zum Mehrklang, zur Mehrstimmigkeit führte, was einen Bruch zur übrigen Welt darstellte. ${ }^{37}$ Noch schärfer normativ versucht Friedrich A. Schmid Noerr, die europäische Kunst in der Gegenwart der

34. E. MIKA, Reich und Abendland, in: Europa - Kontinent der Jugend, op.cit., S.14-19, S.18 f.

35. F.A. SCHMID NOERR, Europa und die Bildende Kunst, in: Europa-Kontinent der Jugend, op.cit., S.21-33, hier: S.22.

36. Ibid., S.29.

37. A. OREL, Musik Europas, in: Europa - Kontinent der Jugend, op.cit., S.38-41, hier: S.38. 
vierziger Jahre des 20. Jahrhunderts zu einer Rückbesinnung auf ältere europäische Traditionen aufzurufen:

„Besinnung auf den abgründigen Unterschied zwischen blutvoll eigenwilliger, repräsentativer Genialität und blutleer abstrakt räsonierenden, verwaschenen Kollektivismen eines international sich gebärdenden Kunstjargons“ “. ${ }^{38}$

Das Europäische in der Kunst wird dabei einerseits als etwas Gemeinsames identifiziert, gleichsam jedoch auf einer qualitativ bewertenden Ebene als überlegen skizziert, wodurch andere Kulturen aus einer ethnozentrischen Perspektive heraus Leistungen abgesprochen werden..$^{39}$ Eines der europäischen Ziele würde daher in der Abschottung und ehrfürchtigen Rückbesinnung (u.a. auf die „volkseigene Seelensprache“) bestehen, da der „Internationalismus“ in der Kunst Liberalismus, Individualismus und Eklektizismus, somit nur Beliebigkeit gebracht und zur „Auflösung der europäischen Wesens- und Stilgemeinschaft" geführt hätte. ${ }^{40}$

Es ist auffällig, dass die Tagungspublikation die historische Dimension so stark in den Mittelpunkt stellt, da sich die Reden auf der Gründungsfeierlichkeit selbst und die Presseberichte dieses Aspektes nur vage annehmen. Einzig eine im Rahmen des Festprogramms stattfindende Ausstellung in der Nationalbibliothek (,Dokumente europäischer Geschichte") wendet sich Teilen der europäischen Vergangenheit zu, die anhand von in Wien archivierten Akten und Dokumenten ausgestaltet wurde (u.a. preußisch-österreichischer Staatsvertrag über die dritte Teilung Polens 1795, Akten des Wiener Kongresses 1815, Akten des Berliner Kongresses 1878). Bei der Eröffnung wurde seitens der Nationalbibliothek die Rolle Wiens als „Bollwerk Europas“ beschworen und auf die aktuelle Verteidigung der alten Kultur verwiesen. ${ }^{41}$ Mit der Platzierung eines „Bollwerkes“ für Europa wird deutlich, dass die Organisatoren in Wien durchaus versuchten, einen zu engen national gezogenen Pathos zu vermeiden, wenngleich die Beispiele - auch in den Ausführungen der Wissenschaftler im Tagungsband - meist eine deutsche Perspektive privilegierten und aus ihr heraus damalige Geisteshaltungen zu beeinflussen suchten. $\mathrm{Zu}$ betonen gilt es jedoch nochmals, dass die historische Legitimation jenen Aspekt darstellt, der zwar überproportional stark in der Publikation „Europa - Kontinent der Jugend“ vertreten ist, im realpolitischen Diskurs auf den Veranstaltungen zur Gründung des „Europäischen Jugendverbandes" aber eine untergeordnete Rolle einnahm.

Weitaus präsenter war der antike Mythos. Obwohl es fraglich ist, ob der Raum „Europa“ überhaupt etwas mit dem griechischen Mythos rund um Europa und Zeus, der als Stier auftritt, um diese zu entführen, zu tun hat, ${ }^{42}$ gibt es in der europäischen

\footnotetext{
38. F.A. SCHMID NOERR, op.cit., S.33.

39. Ibid., S.26.

40. Ibid., S.28 ff.

41. AdR, RSTTH Wien, Kt. 53, 278, „Europäischer Jugendverband, Wien 1942“, Pressebüro/ [Rede Nationalbibliothek?]. Vgl. auch VB, Wiener Ausgabe, 15.09.1942, S.3.

42. Vgl. u.a. M. GEHLER, op.cit., S.11.
} 
Kulturgeschichte eine lange Tradition, auf diese Erzählung zu rekurrieren. ${ }^{43}$ Auch die Organisatoren der Gründungsveranstaltungen zum Jugendverband in Wien, bedienten sich dieser Vorstellung als Symbol. Im „Gauhaus“ wurde eine Stuckskulptur von „Europa auf dem Stier“ aufgestellt, auf einer Erinnerungsmedaille, am Cover des Tagungsbandes und auf dem Briefpapier der Organisatoren prangten die beiden Figuren. ${ }^{44}$ Günter Kaufmann, der Propagandachef Schirachs, stellte diesen Mythos als einen, die Grenzen der geschichtlichen Existenz überschreitenden Moment der europäischen Kultur dar, und leitet davon für den Erdteil eine besondere Stellung in der Welt ab: „Denn Gott liebt uns wie einst im Mythos der Vorzeit, und wenn einer das Recht und die Ordnung der Welt zurück gibt, so wird es wieder ein Sohn der Europa sein“! 45 Auch Schirach baut den Mythos, den er als „klassisches Symbol“ des gemeinsamen europäischen Ursprungs wahrnimmt, in seiner Eröffnungsrede ein: „Europa ist mehr als ein Kontinent. Europa ist ein heiliges Wahrzeichen der Menschheit". 46

Doch neben diesen Bezügen auf die vergangene und sogar mythische Zeit Europas war es stärker die Gegenwart und die Zukunft, die als Identitätsfolien angeboten wurden.

\section{Legitimation über die Gegenwart und Zukunft}

Bei der Analyse der historischen Quellen wird schnell deutlich, dass jene Aussagen, die über die Intentionen des „Europäischen Jugendverbandes“ getroffen wurden, nicht nur den Frieden und die inhaltliche Zusammenarbeit der beteiligten Nationen zum Ziel hatten, sondern darüber hinaus einen vielschichtigen normativen Katalog mitlieferten, der argumentativ aus der Gegenwart und den Erwartungen bezüglich der Zukunft abgeleitet wurde.

Durch ein Beschwören der „europäischen Schicksalsgemeinschaft“ im Weltkrieg wird eine eindeutige Wir-Identität kommuniziert, die stark mit der nationalsozialistischen Ideologie (Antibolschewismus, Antiliberalismus, Antisemitismus, Kritik an der Demokratie usw.) verzahnt wurde. Der laufende Krieg diente dabei aber als zen-

43. Vgl. S. SALZMANN (Hrsg.), Mythos Europa. Europa und der Stier im Zeitalter der industriellen Zivilisation, Ellert \& Richter, Hamburg, 1988.

44. W. SCHMALE, Geschichte Europas, S.126; B. DECKER, „Europäischer Jugendkongreß “, Wien 1942, in: S. SALZMANN (Hrsg.), op.cit., S.132-136; M.-L. von PLESSEN (Hrsg.), Idee Europa. Entwürfe zum „Ewigen Frieden “, Henschel Verlag, Berlin, 2003. AdR, RSTTH Wien, Kt. 52, 278 - Presseberichte, vgl. dort Briefpapier des Pressebüros.

45. G. KAUFMANN, Mythos Europa, in: Europa - Kontinent der Jugend, Verlag „Die Pause“, Leipzig, 1942, S.5-8, hier S.8.

46. Der Europäische Jugendverband in Wien gegründet, in: VB, Wiener Ausgabe, 15.09.1942, S.4. 
trales Bindemittel zwischen den Teilnehmernationen. Taras Borodajkewycz ${ }^{47}$ schrieb im Tagungsband dazu:

„Der Sieg gehört demjenigen, der die beherrschenden Ideen der Zeit an seine Fahne heftet. Waren es vor 150 Jahren die Armeen der Französischen Revolution mit ihrem Schlachtruf von Freiheit, Gleichheit und Brüderlichkeit, so sind es heute die grauen Kolonnen des deutschen Heeres und seiner europäischen Waffengefährten. In ihren Reihen steht das Europa, das sich jung und neu fühlt und weiß, daß der nationale Sozialismus das Gesicht des Erdteils bestimmen wird, mit ihnen streiten, wie einst die Walküren, alle guten Geister der großen abendländischen Vergangenheit für eine Zukunft, die nicht nur ein Europa der Macht bringt, sondern auch ein neues Europa des Geistes, das sich der großen Tradition dieses Erdteils, die ihm die Führung der Welt gab, würdig erweist““.48

Und noch eindeutiger formuliert der „Völkische Beobachter“:

„Die Zukunft Europas wird auf den Schlachtfeldern dieses Krieges entschieden. Der Sieg der jungen Völker ist die Vernichtung des kulturfeindlichen Bolschewismus und die Ablösung der britischen Weltherrschaftsepoche. Dieser erkämpften Zukunft unseres Kontinents Inhalt und blutvolles Leben zu geben, sie dem Gesetz der Einheit unterzuordnen, ist der erste Beschluss des Europäischen Jugendverbandes““ ${ }^{49}$

Um diese Verpflichtung der Jugend zu unterstreichen, wird auf das „Blut der Soldaten“ 50 verwiesen. Die Heldenehrungsfeierlichkeit im Rahmen der Gründungstagung am Wiener Heldenplatz unterstreicht diese Argumentation auf emotionaler und inszenierter Ebene. Die kroatische Zeitung „Nova Hrvatski“ sieht anlässlich dieser Ehrung in der Gründung des Europäischen Jugendverbandes den „Beweis, dass die Jugend Europas bereit ist, ihr Leben im Kampf zu opfern und damit die Freiheit des europäischen Kontinents zu erringen". ${ }^{51}$ Auch slowakischen Zeitungen sehen im Krieg das zentrale Moment für den europäischen Zusammenschluss. In einem Bericht des Deutschen Nachrichtenbüros heißt es:

„Die Jugend des neuen Europa, schreibt ,Slowak', hat sich eng zusammengeschlossen, um der gewaltigen Gefahr, durch die die europäische Zivilisation vom jüdischen Bolschewismus und von den westlich jüdisch-plutokratischen Imperialisten bedroht wird, zu steuern". 52

Auch die tschechische Rezeption verläuft ähnlich, indem der „Europäische Jugendverband“ als ,ein Symbol der Neuordnung und des unverbrüchlichen Glaubens der

47. Taras Borodajkewycz wird in den 1960er Jahren wegen seiner NS-Vergangenheit und seinem auch nach 1945 offen gelebten Antisemitismus zu einem der ersten Fälle der österreichischen Vergangenheitsbewältigung, welche die 68er-Bewegung sanft einläutete. Vgl. P. EBNER, K. VOCELKA, Die zahme Revolution. '68 und das was davon blieb, Ueberreuter, Wien, 1998, S.59 ff.

48. T. BORODAJKEWYCZ, Europa. Die Revolutionen, in: Europa - Kontinent der Jugend, Verlag „Die Pause“, Leipzig, 1942, S.53-54.

49. Der Ruf der Jugend, in: VB, Wiener Ausgabe, 14.09.1942, S.1 f.

50. $V B$, Wiener Ausgabe, 14.09.1942, S.2.

51. BA Berlin, R/901, 58165 - DNB, Nr. 262/Eigendienst, 19.09.1942, Blatt 78.

52. BA Berlin, R/901, 58165 - DNB, Nr. 257/Eigendienst, 19.09.1942, Blatt 68. 
Jugend an den Endsieg der neuen Völker, die für eine gerechte soziale Ordnung streiten", interpretiert wird. ${ }^{53}$

Diese Überbetonung des Weltkrieges als Auslöser der Verbandsgründung wird auch von nicht beteiligten Staaten wahrgenommen. In der Türkei wird daher u.a. davon ausgegangen, dass der „Kongress der europäischen Jugend“ ein Vorwand wäre, um eine „Konferenz der Vasallenstaaten“ in Wien abzuhalten, um auf die Teilnehmer Druck hinsichtlich der Mobilisierung für die Ostfront auszuüben:

„Die Deutschen konnten eine solche Konferenz natürlich nicht offiziell nennen, weil das die Veröffentlichung von Berichten über seine [sic] Arbeit zur Folge haben würde und der ganzen Welt die äusserste Unbeständigkeit und die Widersprüche in der ,neuen europäischen Ordnung" vor Augen führen würde“.

In Verkennung der politischen Ämter von Aldo Vidussoni und Renato Ricci wurde gezeigt, dass die „Delegierten“ nichts mit der Jugendbewegung zu tun hätten. ${ }^{54}$

Der laufende Weltkrieg bot auch Gelegenheit, um die ideologische Abschottung Europas gegenüber dem als nicht-europäisch Identifizierten zu begründen. Was anfänglich als innerdeutsches Problem angesehen wurde, nämlich der Kampf gegen die Demokratie und den Bolschewismus, wurde 1942 bereits als Aufgabe auf Europa projiziert. ${ }^{55}$ Die rassistische Ideologie des Nationalsozialismus wurde jedoch nicht zuletzt von Baldur von Schirach selbst den Gründungsfeierlichkeiten beigemengt. In der Begrüßungsrede vom 14. September 1942 ergeht er sich in antisemitischen Hasstiraden, welche Michael Wortmann als verbalen Kraftakt versteht, um der Veranstaltung doch noch die erhoffte Publizität zu verschaffen. ${ }^{56}$ Schirach hebt in seiner Rede hervor:

„Jeder Jude, der in Europa wirkt, ist eine Gefahr für die europäische Kultur. Wenn man mir den Vorwurf machen wollte, daß ich aus dieser Stadt, die einst die europäische Metropole des Judentums gewesen ist, Zehntausende und aber Zehntausende von Juden ins östliche Ghetto abgeschoben habe, muß ich antworten: Ich sehe darin einen aktiven Beitrag zur europäischen Kultur". .57

Die bei der Rede verarbeiteten antisemitischen Konstruktionen dürften aber vermutlich mehreren Zwecken gedient haben. So vermutlich auch einem Sichtbarmachen

53. BA Berlin, R/901, 58165 - DNB, Nr. 258, 15.09.1942, S.28. Vgl. ad Dänemark und Slowakei auch DNB, Nr. 259, 16.09.1942, S.7. Ad Niederlande vgl. AdR, RSTTH Wien, Kt. 53, 278 - Presseberichte.

54. BA Berlin, R/901, 58165 - Transocean Berlin, I-Dienst, 59, 17.09.1942. Tass zum Wiener Jugend Kongress. - Vidussoni war zwischen dem 26.12.1941 und 19.04.1943 Generalsekretär der Faschistischen Partei. Ricci war zwischen dem 31.10.1939 und 06.02.1943 Ministero delle Corporazioni. Die Anwesenheit dieser beiden faschistischen Politiker verdeutlicht den höheren Grad an Wichtigkeit, welcher den Gründungsfeierlichkeiten seitens des faschistischen Italien beigemessen wurde.

55. „Demokratie, das ist die Welt von gestern, die Welt des angelsächsischen Liberalismus, die Welt der französischen Ideen von 1789, die Welt des atlantischen Imperiums unter Niederhaltung der Mitte Europas“. - T. BORODAJKEWYCZ, op.cit., S.53. Vgl. auch Der Ruf der Jugend, in: VB, op.cit.

56. M. WORTMANN, op.cit., S.213.

57. Der Europäische Jugendverband in Wien gegründet, in: VB, Wiener Ausgabe, 15.09.1942, S.2. 
seiner „Leistungen“ gegenüber einem Auftrag, den Schirach von Hitler erhielt und die Deportation der Juden aus der Stadt zum Inhalt hatte. ${ }^{58}$ Im Zusammenhang mit dem Jugendverband erkennt Schirach zwar selbst, dass sein antisemitischer Exkurs in der Rede eine „Abschweifung“ darstellte, betonte jedoch mit dem Beispiel auch: „Wir sind eben durch unseren nationalen Charakter bestimmt. Der Jude aber ist international" ${ }^{59}$ Damit wird indirekt, wie noch zu zeigen sein wird, auf die strukturelle Zusammenarbeit im Verband - als Zusammenschluss einzelnen Nationen - verwiesen. Das Jüdische wird bei Schirach darüber hinaus als negativer Einfluss auf die Jugend begriffen und mit bekannten Beispielen aus der NS-Propaganda untermauert (u.a. über die Revue, das Kino, die Sexualmoral). Gustav Memminger hatte bereits in seinem Auftaktartikel im „Völkischen Beobachter“ zum Beginn der Veranstaltung auf diesen Umstand verwiesen:

„Der Jude ist der ewige Feind der Jugend. Daß seine Einflüsse zur restlosen Vernichtung gelangen, dafür bürgt die Zusammenarbeit dieser Jugend, die ehrfurchtsvoll zu den völkischen Eigenarten und zum völkischen Brauchtum ihres Landes sich bekennt. In der Wahrung des völkischen Eigenlebens und in der Achtung des religiösen Bekenntnisses, das dem obersten Grundsatz der Einheit und der Kultur Europas unterworfen bleibt, offenbart sich die Freiheit des neuen Europas". ${ }^{60}$

Vergleicht man diese ideologische Stoßrichtung mit der Zusammenstellung von Presseberichten aus den unterschiedlichen teilnehmenden Ländern für das Büro Schirach, zeigt sich, dass nur ganz wenige Meldungen der europäischen Presse zum „Europäischen Jugendverband“ der antisemitischen Argumentation folgten. ${ }^{61}$ Vielmehr wurde der „Europäische Jugendverband“ als Hoffnung interpretiert, um als eigenes Land einen Rang im „neuen Europa“ zu erlangen. Die tschechische „Narodni stred" betont:

„Die Tatsache, daß auch Vertreter der tschechischen Jugend im Rahmen der deutschen Abordnung an der Gründung des neuen Europäischen Jugendverbandes teilnehmen konnten, sei ein weiteres Zeichen dafür, daß der Weg mit dem Reich dem tschechischen Volk die Tür zu Europa öffne". ${ }^{\circ}$

Aber auch in anderen Pressemeldungen kann man erkennen, dass die teilnehmenden Staaten, die ganz unterschiedlich durch den Nationalsozialismus dominiert waren, über den Europäischen Jugendverband ein kameradschaftliches Einvernehmen anstrebten. ${ }^{63}$

58. M. WORTMANN, op.cit., S.192.

59. Der Europäische Jugendverband in Wien gegründet, in: VB, op.cit.

60. Der Ruf der Jugend, in: VB, op.cit.

61. Vgl. u.a. AdR, RSTTH Wien, Kt. 53, 278, Presseberichte. Lidove Listy, Prag (19.09.1942) oder Nova hrvatska, Agram (16.09.1942?).

62. BA Berlin, Nr.258, S.28, 15.09.1942. Vgl. dazu auch AdR, RSTTH Wien, Kt. 53, 278, Presseberichte, Narodni Stred, Prag (15.09.1942). Hier zeigt sich auch inwieweit die NS-Verwaltung unterschiedliche Paraphrasierungen von Presseberichten bei der Wiedergabe der Inhalte vornimmt!

63. AdR, RSTTH Wien, Kt. 53, 278, Presseberichte - z.B. Nieuwsbron, Den Haag (16.09.1942). 
Es ist eigentlich nicht weiter verwunderlich, dass man versuchte, Versatzstücke der NS-Propaganda auch innerhalb des Europäischen Jugendverbandes zu platzieren. Die antisemitische und antibolschewistische Ausrichtung stieß jedoch bei den Teilnehmerstaaten nicht auf den gleichen Widerhall wie die Idee von kameradschaftlich vereinten Staaten und einer gleichberechtigten Zusammenarbeit, wie dies im nächsten Abschnitt dieser historischen Betrachtung als Ziel des Verbandes herausgearbeitet wird.

\section{Struktur der Zusammenarbeit im „Europäischen Jugendverband“}

Da, wie bereits oben angemerkt, kein politisches Positions- oder Strategiepapier zur Konzeption des „Europäischen Jugendverbandes“ bisher in den Archiven aufgefunden werden konnte, muss man, will man die Struktur des europäischen Zusammenschlusses herausarbeiten, auf konzeptionelle Äußerungen der Beteiligten zurückgreifen bzw. die Strukturen des ersten Treffens heranziehen.

Es überrascht, dass der „Völkische Beobachter“ zum Auftakt der Gründungsfeierlichkeiten am 14. September 1942 am Titelblatt die „Gleichberechtigung und gegenseitige Anerkennung der Jugend der europäischen Nationen“ des „Europäischen Jugendverbandes" hervorhebt, um dessen Basis zu charakterisieren. ${ }^{64}$ Demokratische Strukturen waren damit jedoch nicht verknüpft. Das nationalsozialistische Deutschland, in diesem Fall vertreten durch Baldur von Schirach, war jene Macht, welche die Ämter verteilte. Im Minutenprogramm der Veranstaltungsplanung wurde hinsichtlich der Ernennung des Präsidiums des Verbandes festgehalten:

„Der Reichsleiter [Schirach] begibt sich nach der Begrüßungsfanfare nach dem Platz des Ehrenpräsidenten, leitet die Eröffnungssitzung ein und begrüßt alle Reichsleiter für die Jugenderziehung der NSDAP und Reichsstatthalter in Wien, die Abordnungen der europäischen Jugend und ernennt Exzellenz Vidussoni und den Reichsjugendführer der NSDAP und Jugendführer des Deutschen Reichs Artur Axmann zu Präsidenten des Europäischen Jugendverbandes". .5

Nichts wurde dabei dem Zufall überlassen, auch nicht die daran anschließende Ernennung der Ehrenpräsidenten Schirach und Ricci. Die anwesenden Vertreter/-innen der Nationen durften durch Erheben von den Plätzen und durch Beifall diesen Vorgang bestätigen.

Nachdem der Führungsanspruch auf diese Weise durch Vertreter der „Achsenmächte" abgesichert worden war, verkündete Schirach in seiner Rede die Möglichkeiten der politischen Partizipation innerhalb des Verbandes:

„Der Europäische Jugendverband ist keine Schöpfung der Demokratie. Es würde dem Geist der Jugend und unserer ganzen bisherigen Zusammenarbeit widersprechen, wenn

64. VB, Wiener Ausgabe, 14.09.1942, S.1, Aufmarsch der europäischen Jugend.

65. AdR, RSTTH Wien, Kt. 53, 278 - Reichsstatthalterei Minutenprogramm. 
wir nach dem parlamentarischen Zahlensystem verfahren würden, das heißt das Gewicht der einzelnen Jugendorganisationen im Führerring abhängig machen würden von der Zahl der Mitgliederorganisationen. In diesem Gremium hat jede nationale Jugend, die wir einladen, sich dem internationalen Jugendverband anzuschließen, ob sie nun einem kleinen oder großen Volk angehört, Sitz und Stimme. [...] Die Mitglieder des Europäischen Jugendverbandes sind grundsätzlich gleichberechtigt". 66

Dem italienisch-deutschen Präsidium wird dabei eine Sonderstellung eingeräumt, da es gemeinsam Beschlüsse fällen könnte, die an die übrigen Mitglieder weitergeleitet werden würden.

Um die gepriesene Gleichheit auf der Gründungsveranstaltung in Wien sofort sichtbar zu machen, wurde seitens der Veranstalter veranlasst, dass den unterschiedlichen Arbeitsgruppen je ein anderer Jugendführer aus den teilnehmenden Ländern vorsaß. Doch auch dort war der Spielraum begrenzt. Die Organisatoren hatten bereits im Vorfeld für die vierzehn unterschiedlichen Arbeitsgemeinschaften inhaltliche $\mathrm{Pa}$ piere vorbereitet. ${ }^{67}$

Ziel des Europäischen Jugendverbandes war es nicht, alle Jugendorganisationen Europas in gleicher Weise zu organisieren. Man ging vielmehr davon aus, dass Erziehung eine nationale Angelegenheit wäre. Die Aufgabe des Verbandes wäre es demnach, ein Bewusstsein der europäischen Zusammengehörigkeit der jungen Generation zu festigen und zu stärken. In diesem Zusammenhang betonte man mehrmals, dass im Verband „selbstständige und volksbewusste Jugendorganisationen“ zusammenarbeiten würden, weshalb sich ein Einmischen in die inneren Verhältnisse der Länder ausschließen würde. Dezidiert wurde von Schirach hervorgehoben, dass der „Europäische Jugendverband“ alle paneuropäischen Bestrebungen ablehne. Durch die Betonung der nationalen Eigenart innerhalb des Verbandes wurde Europa als „Strahlenbündel nationaler Kräfte“ (Schirach) interpretiert. Nach Schirach wollte man damit eine ,Vermanschung und Vermischung dieser nationalen Kräfte“ verhindern, wie man sie in den als kulturell steril klassifizierten USA zu erkennen glaubte. ${ }^{68}$ Günter Kaufmann versuchte im Oktober 1942 sogar eine Neuinterpretation bezüglich der Begegnung der europäischen Völker vorzulegen, die jedoch vermutlich niemals eine breite Öffentlichkeit erreichte, aber dennoch Einblicke in den Kreis um Schirach erlaubt:

„Der Europäische Jugendverband mit seinen Prinzipien macht sich das Gesetz der kämpfenden Front Europas zu eigen. Er anerkennt nur die Leistung für die europäische Völkerfamilie. Es ergibt sich daraus, dass das nationalsozialistische Reich keine Klassifizierung der Völker nach ihrer Rassezugehörigkeit vornimmt, sondern in Würdigung der völkischen Eigenart nur ihren Einsatz für die europäische Gemeinschaft sieht" 69

66. VB, Wiener Ausgabe, 15.09.1942, S.1, Der Europäische Jugendverband in Wien gegründet.

67. AdR, RSTTH Wien, Kt. 53, 278. - Zentralbüro Schirach, Europäisches Jugendwerk, Reden, Presse, etc. zur Gründungstagung.

68. VB, Wiener Ausgabe, 15.09.1942, S.4, Der Europäische Jugendverband in Wien gegründet - besonders Rede Schirachs zur Gründung des Verbandes.

69. AdR, RSTTH Wien, Kt. 53, 278. - Presseberichte - „Wille und Macht“, Berlin Günter Kaufmann (0ktober 1942). 
Diese für eine europäische Zusammenarbeit notwendige Voraussetzung in der Begegnung der europäischen „Völker“, stand jedoch in eindeutigem Gegensatz zu anderen prominent positionierten Standpunkten, die unter dem Nationalsozialismus Verbreitung fanden (u.a. Abwertung der Slawen durch die SS). ${ }^{70}$

Man gab sich Mühe, den Verband als etwas genuin Neues zu präsentieren. Die stark spürbare Abgrenzung einer für alle europäischen Völker geltende Erziehungsarbeit richtet sich dabei primär gegen die britische Pfadfinderbewegung (,scouts"). Axmann betont bei der Schlussveranstaltung des einwöchigen Programms:

\begin{abstract}
„Der Europäische Jugendverband dient keinem Weltstaat. Die europäischen Jugendführer sind bemüht über ihre eigenen Nationen hinaus einer wahren Volksgemeinschaft in der Raumeinheit Europa zu dienen. Mit dem Europäischen Jugendverband ist durch Idee und Tat der Weltverband der Scouts überwunden. Für die neue Ordnung unseres Erdteils kämpfen heute gemeinsam unsere Soldaten. Europa wird nicht durch den Vertrag, der mit kühler Tinte unterschrieben ist, gestaltet, sondern durch das Blut unserer Besten ". ${ }^{71}$
\end{abstract}

\title{
Schlussfolgerung
}

Das von Baldur von Schirach und seinen Mitstreitern in Wien erdachte Europa, welches im Rahmen des „Europäischen Jugendverbands“ ausgestaltet wurde, ist eindeutig ein Europa von Einzelstaaten, die sich entgegen älterer Modelle der Nachkriegszeit nicht zu einer Union zusammenschließen, sondern eine sehr lockere inhaltliche Zusammenarbeit auf dem Gebiet der Jugenderziehung etablieren wollten. Eine Einmischung von außen in innerstaatliche Angelegenheiten sollte in diesem Gefüge als Zwangsakt abgelehnt werden, um die Idee der „Volkseigenart“ nicht zu unterlaufen. Aus diesem Grund könnte man die Motive der vielen Staaten und ihrer entsandten Staatsjugend, die oft in einer Abhängigkeit oder in einem engen Nahverhältnis zum NS-Staat standen, auch darin erkennen, dass sie die Chance auf die Rehabilitierung einer gewissen (nationalen) Souveränität innerhalb der Gemeinschaft witterten. Die Problematik der politischen Verstrickung der anwesenden „Völker“ untereinander, die zwar das Moment des Weltkriegs einte, deren politische Selbstständigkeit angesichts der europäischen Mächteverhältnisse aber nicht gegeben war, wurde auf der Gründungsveranstaltung jedoch verschwiegen. Der realpolitische Spielraum bzw. der emotionale Zugang zum „Europäischen Jugendverband“ der einzelnen teilnehmenden Staaten müsste daher in einer eigenen Studie noch jeweils genauer überprüft werden.

Aus deutscher und italienischer Perspektive - zumindest seitens der Leiter der Jugendorganisationen - wurden sicherlich primär die Führungsrolle innerhalb des Jugendverbandes und der erfolgreiche Export von ideologischen Modellen in andere europäische Länder wahrgenommen. Was Schirach und seine Gefolgsleute in Wien

70. W. Burgdorf, op.cit., S.193.

71. VB, Wiener Ausgabe, 20.09.1942, S.3, Die Ansprache des Reichsjugendführers. 
ignorierten, war die Tatsache, dass die „Achse Berlin-Rom“ zu diesem Zeitpunkt bereits eine seltsame Patina angesetzt hatte und nicht von allen in der NSDAP als der optimale außenpolitische Weg angesehen wurde, wodurch der von den Initiatoren vermutlich erhoffte innerparteiliche Erfolg ausblieb. Der Verlauf des Weltkrieges verunmöglichte es, den Jugendverband inhaltlich und organisatorisch weiterzuentwickeln. Dies wäre aber vor allem in jenen Bereichen interessant gewesen, in denen bei den Feierlichkeiten in Wien eine tendenzielle Überwindung von Teilbereichen des nationalsozialistischen Rassismus als vorläufiges Zugeständnis auszumachen war bei gleichzeitigem Fortschreiben von antisemitischen und nunmehr eurozentrischen Ideologemen, die das „Völkische“ und die nationale, nun europäisch gewendete Überlegenheit gegenüber dem Rest der Welt propagierten. Damit versuchte man auch jene Legitimität des „Europäischen Jugendverbandes“ aufzubauen, der für eine solche Neugründung von Nöten war. Historische Modelle aus unterschiedlichen Bereichen (Politik, Malerei, Musik, etc.) sollten die Tragfähigkeit der europäischen Kultur hervorheben. Dass man dabei das europäisch Gemeinsame betonte, welches auch in der „Schicksalsgemeinschaft" des laufenden Krieges ausgemacht wurde, sowie auf einen schöngeistigen Europa-Mythos rekurrierte, zeugt von einem umfassenden Versuch Argumente zu liefern.

Betrachtet man die Gründungsfeierlichkeiten und das dort konstruierte Europagebäude jedoch aus einer europäischen Perspektive, zeigt sich rasch, dass die Vertreter des nationalsozialistischen Deutschlands ein Interpretationsvorrecht besaßen und allen anderen beteiligten Staatsjugendvertretern - selbst den italienischen „Achsenfreunden" - eine Statistenrolle zu Teil wurde. Aus den in Wien archivierten Materialien zu den Vorbereitungen und der Umsetzung der Gründungsfeierlichkeiten geht hervor, dass die dort geschaffenen Vorstellungswelten zum „Europäischen Jugendverband" eine Schöpfung des Dunstkreises um Baldur von Schirach waren. 